\title{
PRONÓSTICO DEL CAUDAL DEL RÍO CRAVO SUR, SECTOR DEL MORRO - PUENTE LA CABUYA, DEPARTAMENTO DE CASANARE, COLOMBIA
}

\author{
FLOW FORECAST OF THE CRAVO SUR RIVER, \\ SECTOR OF EL MORRO - LA CABUYA BRIDGE, \\ CASANARE DEPARTMENT, COLOMBIA
}

\author{
1 Linson Javier Higuera Infante \\ 2 William Rojas Vergara \\ ${ }^{1}$ Ingeniero Civil, Fundación Universitaria Internacional del Trópico Americano UNITROPICO, Yopal Casanare, Colombia \\ 2 Ingeniero Civil, Esp. Educación Ambiental. Docente Investigador, Fundación \\ Universitaria Internacional del Trópico Americano UNITROPICO, Yopal Casanare, Colombia \\ Javi.higuera0626@gmail.com \\ ${ }^{2}$ Williamrojas@unitropico.edu.co
}

\section{RESUMEN}

Colombia presenta una gran red hidrográfica en la región de los llanos orientales donde el Instituto de Hidrología, Meteorología y Estudios Ambientales IDEAM cuenta con estaciones hidrometeorológicas capaces de llevar registros de lluvia en el tiempo, siendo estas necesarias para la realización de estudios que permitan efectuar seguimiento a esta red y así fundamentar la toma de decisiones en sectores que se ven afectados por las variaciones climáticas que se presentan. De acuerdo con la situación mencionada, se pretende pronosticar el caudal circundante al Municipio de Yopal y obtener elementos que sirvan de base para prever y minimizar riesgos especialmente al sector del agro colombiano el cual, es característico de esta región. Se plantea un estudio de caso del caudal en la cuenca media del Río Cravo Sur en una serie temporal de periodicidad mensual desde el año 1975 hasta el 2014, luego se realiza un análisis exploratorio de datos el cual, permite visualizar los estadísticos descriptivos que sirven para evaluar el comportamiento general de la serie, posteriormente se analiza la misma con el fin de determinar el modelo más apropiado para el pronóstico.

Como resultado, se determinó que, a lo largo del tiempo, los caudales tienden levemente a aumentar, pero sin generar riesgos superiores a los que en la actualidad está expuesta la región especialmente en épocas de constantes Iluvias; esta estimación se ajusta a las características propias de la estación y depende única y exclusivamente de su historia. 
Palabras clave: estaciones hidrometeorológicas, cuenca hidrográfica, Orinoquía, imputación de datos, análisis exploratorio, estudio de caso.

\section{ABSTRACT}

Colombia has a large hydrographic network in the region of Eastern Plains, where the Institute of Hydrology, Meteorology and Environmental Studies (IDEAM) has hydrometeorological stations than can store rainfall records over time. They are required for develop studies that allows monitoring this network, thus providing a base for decision-making in sectors that are affected by the climatic variations. In accordance with this situation, it is intended to forecast the flow surrounding to the municipality of Yopal and obtain useful elements to anticipate and minimize risks, especially to the Colombian agricultural sector, which is characteristic of this region. A case study of flow in the middle basin of Cravo Sur River was proposed, using a time series of monthly periodicity from 1975 to 2014. After that, an exploratory data analysis was carried out, which allowed the visualization of the descriptive statistics useful to evaluate the general behavior of the mentioned series. Subsequently, the series were analyzed to determine the most appropriate forecasting model.

As a result, it was determined that, as time goes by, flows tend to increase slightly, but without generating risks greater than those currently suffered for this region, especially in times of constant rainfall rates. This estimation is adjusted to the own characteristics of the station studied and depends exclusively on its history.

imputation, exploratory analysis, case study.

Keywords: hydrometeorological stations, hydrographic basin, Orinoquía, data

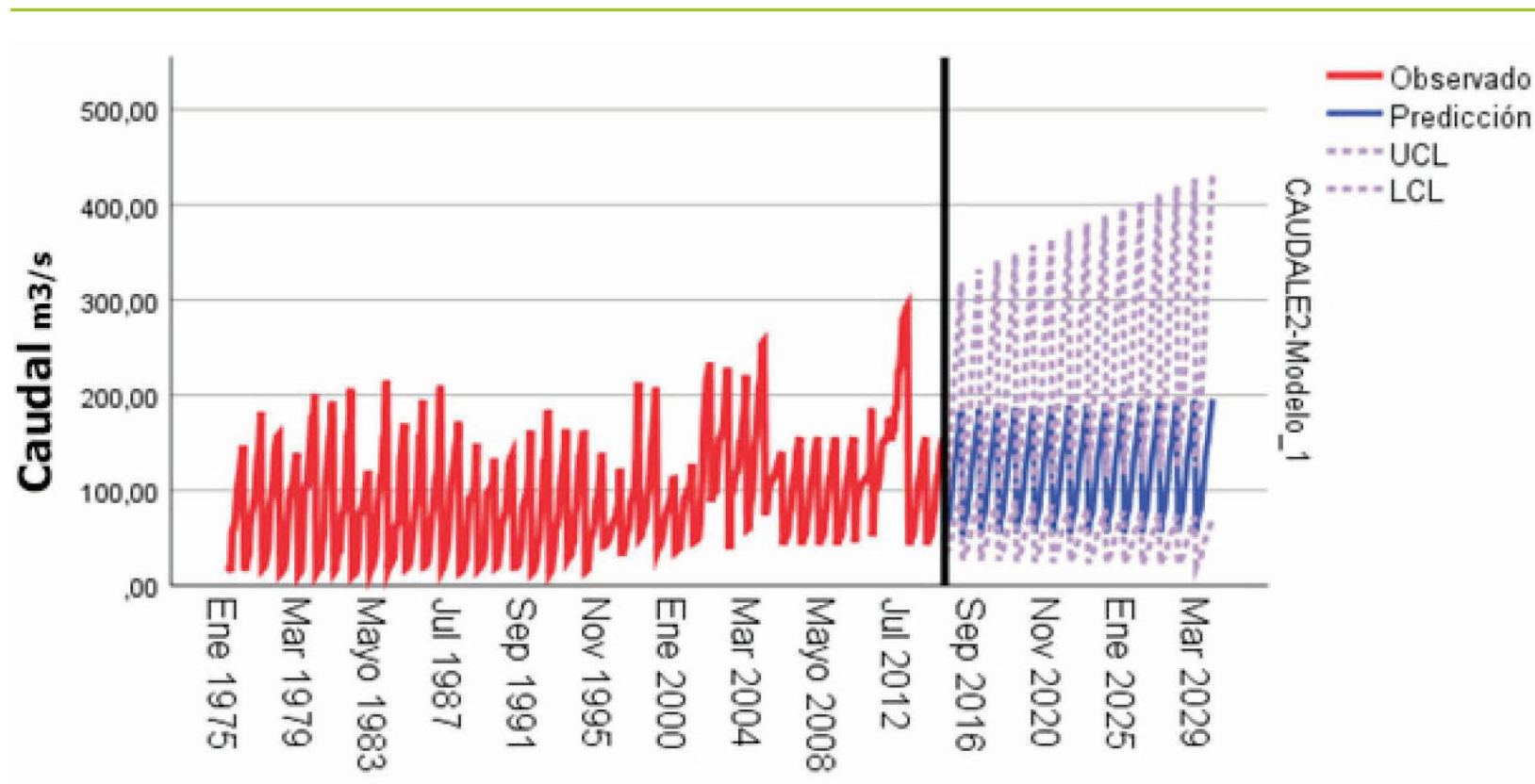

\section{Fecha}

Gráfico 1. Pronostico del caudal 15 años en la cuenca media del Rio Cravo Sur en Yopal Casanare.

Fuente: Datos IDEAM, completación de datos y procesamiento por parte del investigador. 


\section{INTRODUCCIÓN}

En la región de la Orinoquía o Llanos Orientales de Colombia como es conocida comúnmente, se presenta una variación climática en diferentes tiempos del año siendo que para el primer trimestre del mismo se cuenta con un tiempo de sequía como lo demuestran los datos históricos de los últimos 40 años los cuales, fueron suministrados por el Instituto de Hidrología, Meteorología y Estudios Ambientales IDEAM "institución pública de apoyo técnico y científico al Sistema Nacional Ambiental, que genera conocimiento, produce información confiable, consistente y oportuna, sobre el estado y las dinámicas de los recursos naturales y del medio ambiente" (IDEAM, 2014), estos datos, muestran que existen problemas de déficit hídrico en tiempos de sequía, acarreando situaciones fuera de lo normal en el suministro de agua a las poblaciones y el abastecimiento a las actividades del agro incluido la ganadería donde se ven limitadas las mismas por la falta de continuidad y oferta de los productos; por tal razón, se hizo necesario realizar un análisis exploratorio de datos en un periodo comprendido entre el año 1975 y 2014 a la estación hidrometeorológica Puente El Yopal, ubicada en la ciudad de El Yopal-Casanare sector del MORRO - PUENTE LA CABUYA específicamente en la cuenca media del río debido a que en esta se encuentra situada la Ciudad de Yopal Casanare, siendo que es una de las más beneficiadas por este recurso de acuerdo a los datos recopilados por la empresa Enviromental Ingenieros Consultores en la ejecución de contrato de consultoría suscrito con la Corporación Autónoma Regional de la Orinoquia CORPORINOQUIA en el año 2007 con el fin de desarrollar el Plan de Ordenación Y Manejo de la Cuenca del Río Cravo Sur POMCH, allí se especifica que la cuenca es un territorio de estructura rural el cual, del $100 \%$ de su área, solo el 0,26\% alberga los cascos urbanos de los municipios de Labranzagrande, Paya, Pisba, Nunchía y Yopal, donde este último, corresponde al $94.3 \%$ del territorio urbano de la cuenca.

Se pretende pronosticar el caudal del río 15 años y obtener elementos que sirvan de base para la toma de decisiones con el fin de prever y minimizar los riesgos derivados de variaciones extremas en los caudales. En el orden de las ideas anteriores, este documento servirá como herramienta de planeación del territorio.

La situación existente asociada al problema o necesidad, es la presencia de un alto nivel de incertidumbre referente a las condiciones del Recurso Hídrico de las cuencas, específicamente la del rio Cravo Sur, información que viene siendo recopilada por entidades gubernamentales como: Ideam, Ingeominas, IGAC, Gobernaciones, Alcaldías, entre otras.

Algunas características generales de la Cuenca son: el Río Cravo Sur, desde su nacimiento en la cordillera Oriental en territorio del Departamento de Boyacá, específicamente el municipio de Tasco a 3.800 m.s.n.m. entre la Serranía de Peña Negra y el Páramo de Cadilla el cual, se encuentra protegido dentro de los límites del Parque Nacional Natural de Pisba, hasta su desembocadura en el Río Meta, municipio de Orocué Casanare a los 150 m.s.n.m. presenta una importante corriente que baña los municipios de Tasco, Socotá, Gámeza, 
Mongua, Aquitania, Labranzagrande, Pisba y Paya por el Departamento de Boyacá perteneciendo a este un $35.47 \%$ de su extensión total y Támara, Nunchía, Yopal, San Luis de palenque y Orocué por el Departamento de Casanare con el $64.53 \%$ restante; su área total es de 565.113 hectáreas y su forma es oval oblonda a rectangular oblonda, alberga una corriente principal de 205 kilómetros de longitud que transcurre sobre un amplio gradiente altitudinal (CORPORINIQUIA, CORPOBOYACA, \& UAESPNN, 2007).

\section{MATERIALES Y MÉTODOS}

Con el propósito principal de generar un documento que presenten elementos que sirvan de base para la toma de decisiones con el fin de prever y minimizar los riesgos derivados de variaciones extremas en los caudales y a su vez sirva como herramienta de planeación del territorio, se desarrolla una investigación con enfoque cuantitativo debido a que se basa en el análisis exploratorio de una serie de datos históricos con un alcance descriptivo ya que, se analiza información de manera independiente y teniendo en cuenta el siguiente diseño metodológico:

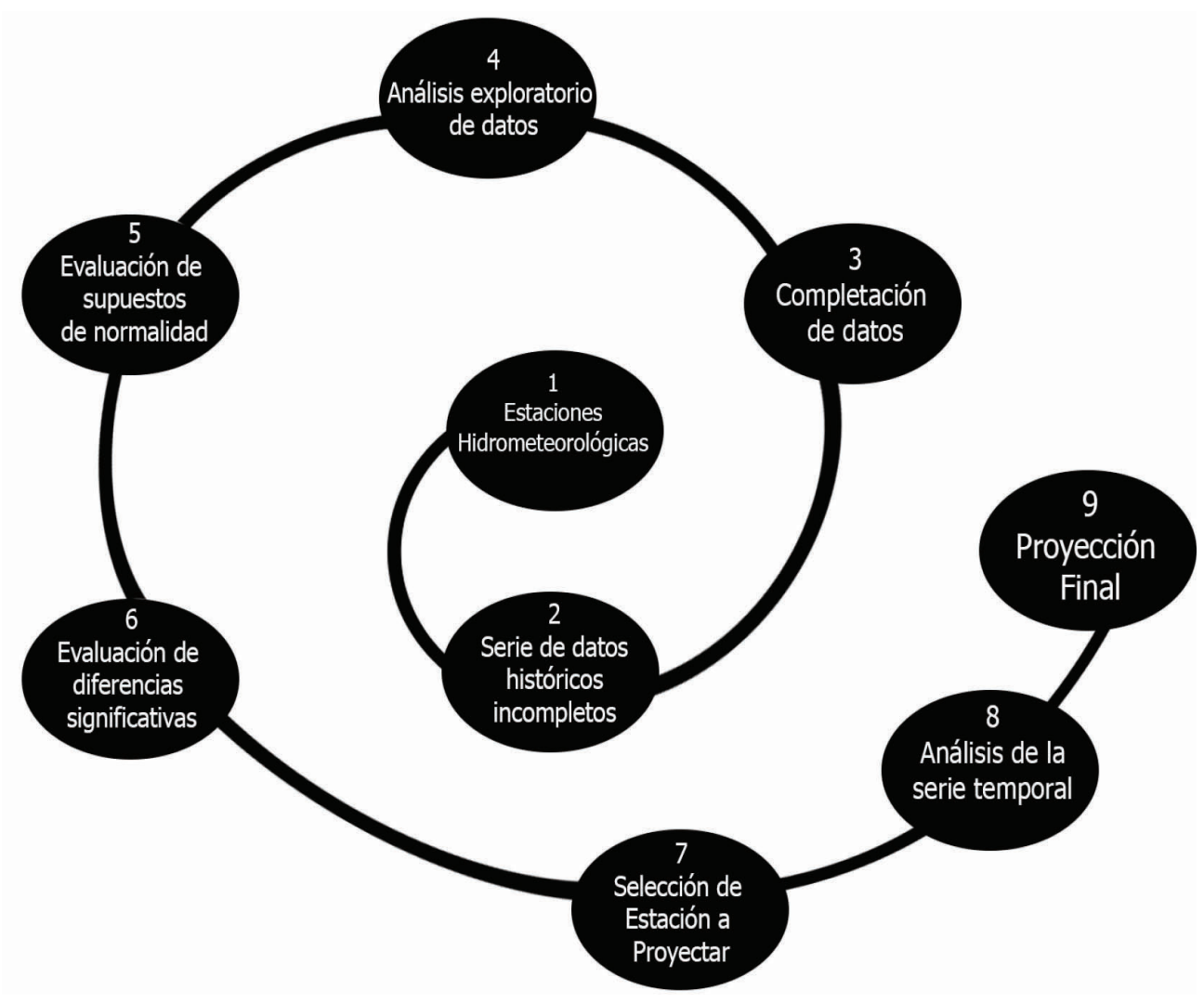

Figura 1. Ruta del proceso metodológico implementado.

Fuente: Investigador 
Localización de la zona de estudio: la cuenca se localiza geográficamente entre los 4041'13" hasta los 5056'37" de Latitud Norte y entre los 71034'09" y 72046'28" de Longitud al Oeste de Greenwich, en conjunto con la influencia que ejerce la Zona de Convergencia Intertropical (ZCIT), condicionan las propiedades físicas de la atmósfera que definen el tiempo y el clima. Actúan también sobre las características climáticas de la cuenca del río Cravo Sur, todas las condiciones físicas que le son propias (forma, orientación, rango de altitudes, topografía, vegetación, etc.) y que producen o modifican los elementos que crean el clima (CORPORINIQUIA, CORPOBOYACA, \& UAESPNN, 2007).

La investigación se desarrolla con los registros históricos de caudales desde el año 1975 hasta el año 2014 provenientes de la estación hidrometeorológica Puente el Yopal operada por el Instituto de Hidrología, Meteorología y Estudios Ambientales (IDEAM) ubicada en el sector comprendido entre el corregimiento El Morro y el Puente La Cabuya el cual, une al municipio de Yopal con el Oriente del Ilano Casanareño, datos que fueron facilitados por este ente gubernamental para fines académicos.

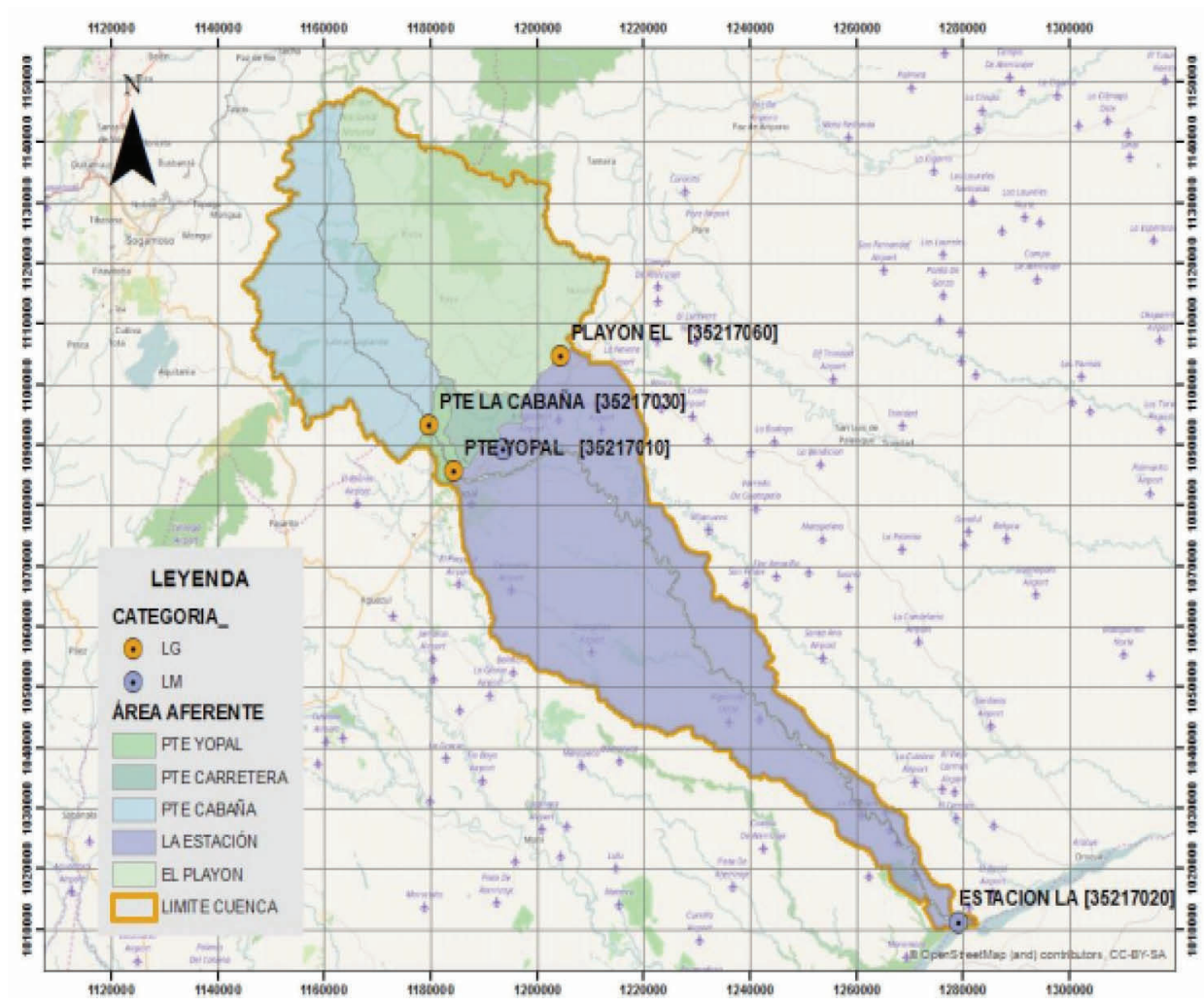

Figura 2. Modelo digital de la Cuenca del Río Cravo Sur y localización de estaciones de caudales.

Fuente de consulta: Actualización Plan de Ordenación y Manejo de la Cuenca del Rio Cravo Sur Código 3521, pág. 36. Consorcio POMCA 201557 
Fase I. Revisión y control de calidad de la serie de datos históricos y análisis exploratorio de datos: el IDEAM, como institución estatal encargada de "Fortalecer la capacidad tecnológica, científica, administrativa y financiera para producir la información hidrológica, meteorológica y ambiental de manera oportuna y con la calidad que requieran la ciudadanía, los sectores públicos y privados del país" (IDEAM, 2014,) fue la entidad facilitadora de los datos históricos de caudales existentes de los últimos 40 años recopilados mediante dos estaciones hidrometeorológicas activas actualmente, ubicadas en el municipio de Yopal, Departamento de Casanare y descritas a continuación:

Tabla 1. Características Estaciones Hidrometeorológicas Cuenca Media Río Cravo Sur

\begin{tabular}{|c|c|c|c|c|c|c|c|c|c|}
\hline \multirow{2}{*}{ No } & \multirow{2}{*}{$\begin{array}{l}\text { Nombre } \\
\text { Estación }\end{array}$} & \multirow{2}{*}{ Código } & \multirow{2}{*}{ Clase } & \multirow{2}{*}{ Categoría } & \multirow{2}{*}{ Lat } & \multirow{2}{*}{ Long } & \multirow{2}{*}{ Alt } & \multicolumn{2}{|c|}{ fecha instalación } \\
\hline & & & & & & & & Inicio & fin \\
\hline 1 & $\begin{array}{l}\text { Puente } \\
\text { La Cabaña }\end{array}$ & 35217030 & Hidrológica & Limnigráfica & 54,379 & $-72,4552$ & 497 & 1979 & 2012 \\
\hline 2 & $\begin{array}{l}\text { Puente } \\
\text { El Yopal }\end{array}$ & 35217010 & Hidrológica & Limnigráfica & 5,369 & $-72,4137$ & 343 & 1974 & 2012 \\
\hline
\end{tabular}

Fuente de consulta: Instituto de Hidrología, Meteorología y Estudios Ambientales IDEAM, http://www.ideam.gov.co/solicitud-de-informacion

Recibidos y tabulados los datos históricos existentes de caudales de las dos estaciones más cercanas a la ciudad de El Yopal descritas anteriormente, logra evidenciarse que no se cuenta con series completas por lo cual, se realiza proceso de imputación de datos para cada una de ellas, optando por completar por el método de los promedios y obteniendo como resultado dos series completas con 480 datos independientes por cada estación.
Se realiza el análisis exploratorio de datos, donde se obtuvo los estadísticos descriptivos básicos, que permitieron realizar una evaluación preliminar de las dos series, teniendo en cuenta la media aritmética, media recortada al 5\%, mediana de cada estación, además de la dispersión y asimetría. Dicho análisis permitió también revisar la existencia o indicios de normalidad. 
Tabla 2. Descriptivos de las estaciones de estudio

\begin{tabular}{|c|c|c|c|}
\hline \multicolumn{3}{|c|}{ Estación } & \multirow{2}{*}{$\begin{array}{c}\text { Estadístico } \\
66,92\end{array}$} \\
\hline \multirow{20}{*}{ CAUDAL } & \multirow{10}{*}{$\begin{array}{l}\text { ESTACION PUENTE } \\
\text { LA CABAÑA }\end{array}$} & Media & \\
\hline & & Media recortada al 5\% & 64,58 \\
\hline & & Mediana & 64,06 \\
\hline & & Varianza & 1728,29 \\
\hline & & Desv. típ. & 41,57 \\
\hline & & Mínimo & 0,66 \\
\hline & & Máximo & 242,80 \\
\hline & & Rango & 242,14 \\
\hline & & Asimetría & 0,75 \\
\hline & & Curtosis & 0,73 \\
\hline & \multirow{10}{*}{$\begin{array}{l}\text { ESTACION PUENTE } \\
\text { EL YOPAL }\end{array}$} & Media & 92,12 \\
\hline & & Media recortada al 5\% & 88,88 \\
\hline & & Mediana & 90,08 \\
\hline & & Varianza & 3298,82 \\
\hline & & Desv. típ. & 57,43 \\
\hline & & Mínimo & 0,00 \\
\hline & & Máximo & 293,30 \\
\hline & & Rango & 293,30 \\
\hline & & Asimetría & 0,67 \\
\hline & & Curtosis & 0,28 \\
\hline
\end{tabular}

Fuente de consulta: Datos IDEAM, completación de datos y procesamiento por parte del investigador.

\section{Fase II. Selección de estación a pronosti-} car: Para determinar si lo conveniente para la investigación era trabajar con la serie de datos de una de las dos estaciones o si por lo contrario se podría promediarlas y pronosticar los caudales con una sola serie se procede a evaluar si existen diferencias significativas en dichas series; previamente se evalúa el cumplimiento del supuesto de normalidad para determinar si se emplearían pruebas paramétricas o no paramétricas; para ello, se emplea la prueba de Kolmogorov - Smirnov rechazándose la hipótesis de que existe presencia de normalidad en ambas series debido a que su significancia se encuentra por debajo del $5 \%$ motivo por el cual, se aplican pruebas no paramétricas para comparar las series y evaluar las diferencias significativas entre ellas.

Tabla 3. Pruebas de normalidad Kolmogorov-Smirnov

\begin{tabular}{l|l|c|c|c}
\multicolumn{1}{|l|}{ Estación } & \multicolumn{1}{|c}{ Estadístico } & gl* & Sig.** \\
\hline \multirow{2}{*}{ CAUDAL } & $\begin{array}{l}\text { ESTACION PUENTE } \\
\text { LA CABAÑA }\end{array}$ & 0,064 & 480 & 0,000 \\
\cline { 2 - 4 } & $\begin{array}{l}\text { ESTACION PUENTE } \\
\text { EL YOPAL }\end{array}$ & 0,078 & 480 & 0,000 \\
\hline
\end{tabular}

Fuente de consulta: Datos IDEAM, completación de datos y procesamiento por parte del investigador.

*Grado de libertad ** Significancia 
Al no contar con presencia de normalidad en las series, se procede a comparar visualmente entre ellas las medianas dentro de los gráficos de caja, los cuales, presentan pocos valores extremos y se evidencia una mediana mayor en la estación Puente El Yopal.

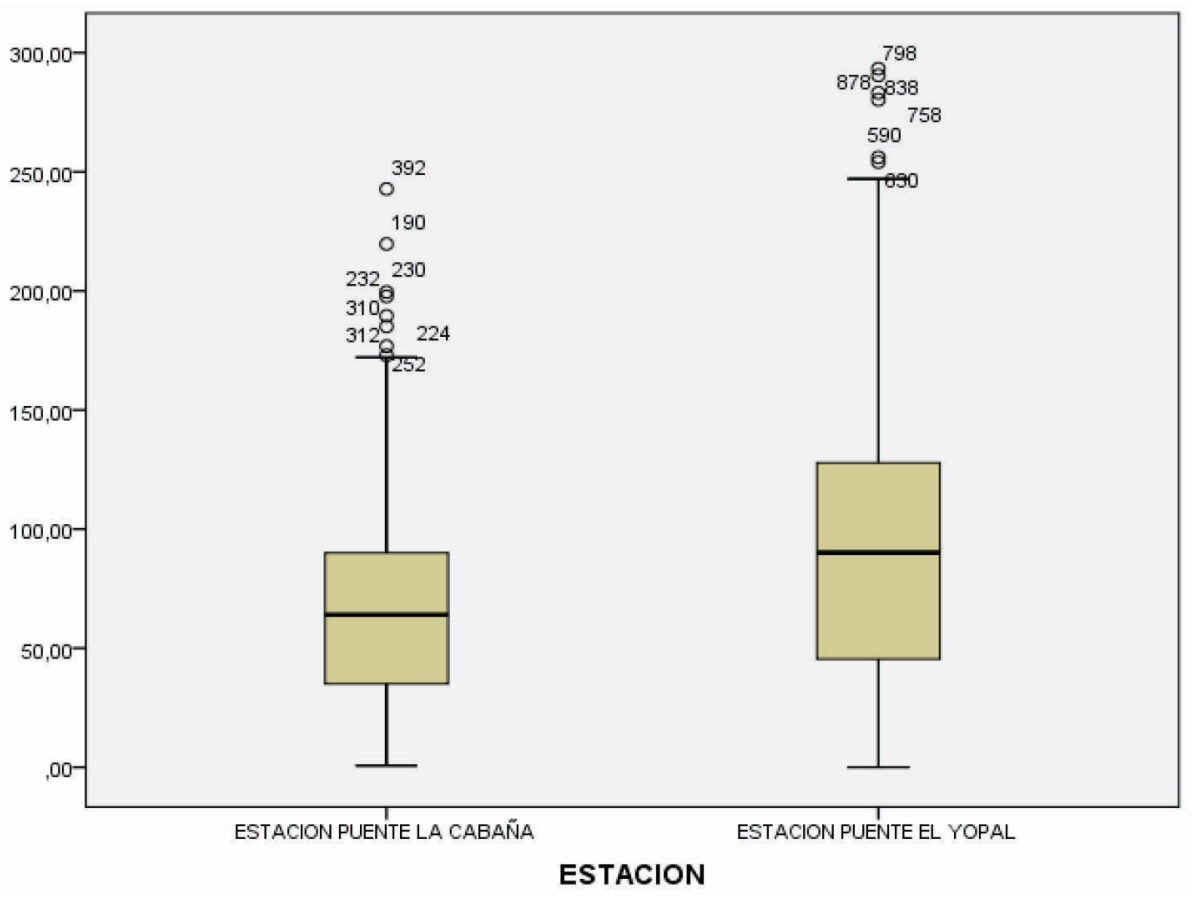

Figura 3. Comparación mediana gráficos de caja Fuente de consulta: Datos IDEAM, completación de datos y procesamiento por parte del investigador.

Estación Puente El Yopal

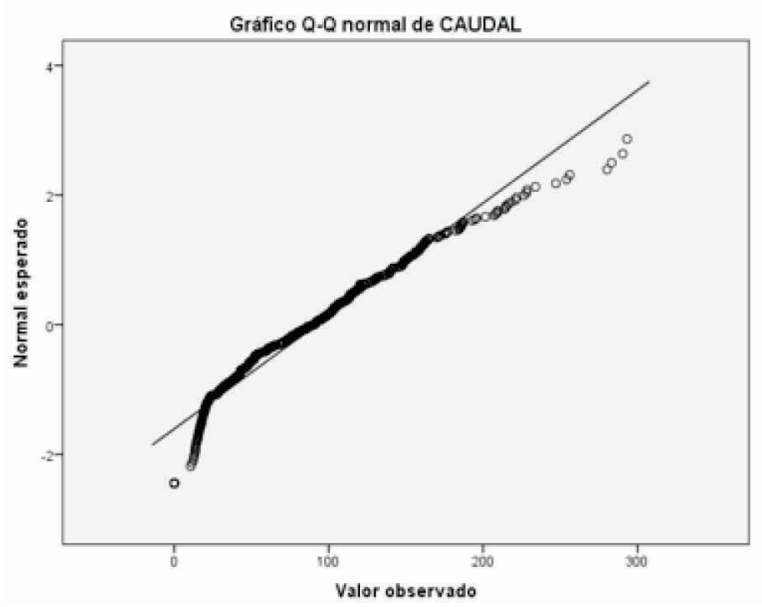

Estación Puente La Cabaña

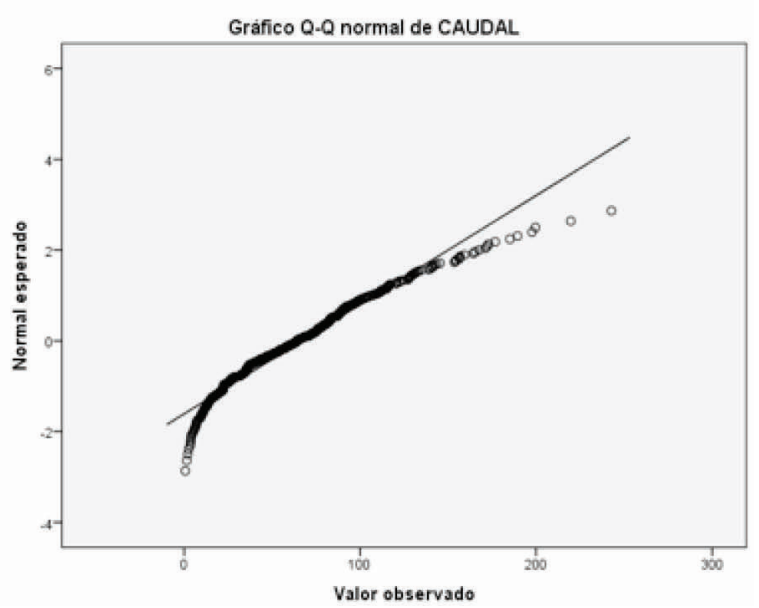

Figura 4. Q-Q normal con tendencia

Fuente de consulta: Datos IDEAM, completación de datos y procesamiento por parte del investigador. 
Basados en los resultados, se aplica la prueba no paramétrica de Mann Whitney evidenciándose diferencias significativas entre las medianas de las series debido a que su significación se encuentra por debajo del 5\%; por lo anterior y de acuerdo al criterio del investigador, se toma la decisión de realizar la pronostico final con la serie de la estación hidrometeorológica Puente El Yopal debido a que esta cuenta con caudales mayores y con el fin de no subestimar el pronóstico.

Tabla 4. Prueba de Mann-Whitney

\begin{tabular}{l|l|c|c|c|}
\hline \multicolumn{5}{c}{ Rangos } \\
\hline \multicolumn{1}{|c|}{ Estación } & N & Rango promedio & Suma de rangos \\
\hline \multirow{3}{*}{ CAUDAL } & \multicolumn{1}{|c|}{ ESTACION PUENTE LA CABAÑ } & 480 & 418,96 & 201098,50 \\
& ESTACION PUENTE EL YOPAL & 480 & 542,04 & 260181,50 \\
\hline & Total & 960 & & \\
\hline
\end{tabular}

Fuente de consulta: Datos IDEAM, completación de datos y procesamiento por parte del investigador.

Tabla 5. Pruebas No Paramétrica de Mann-Whitney

\begin{tabular}{l|c}
\multicolumn{2}{c}{ Estadísticos de contraste } \\
\hline & \multicolumn{1}{c}{ CAUDAL } \\
\hline U de Mann-Whitney & 85658,50 \\
\hline W de Wilcoxon & 201098,50 \\
\hline Z & $-6,87$ \\
\hline Sig. asintót. (bilateral) & 0,000
\end{tabular}

Fuente de consulta: Datos IDEAM, completación de datos y procesamiento por parte del investigador.

\section{RESULTADOS Y DISCUSIÓN}

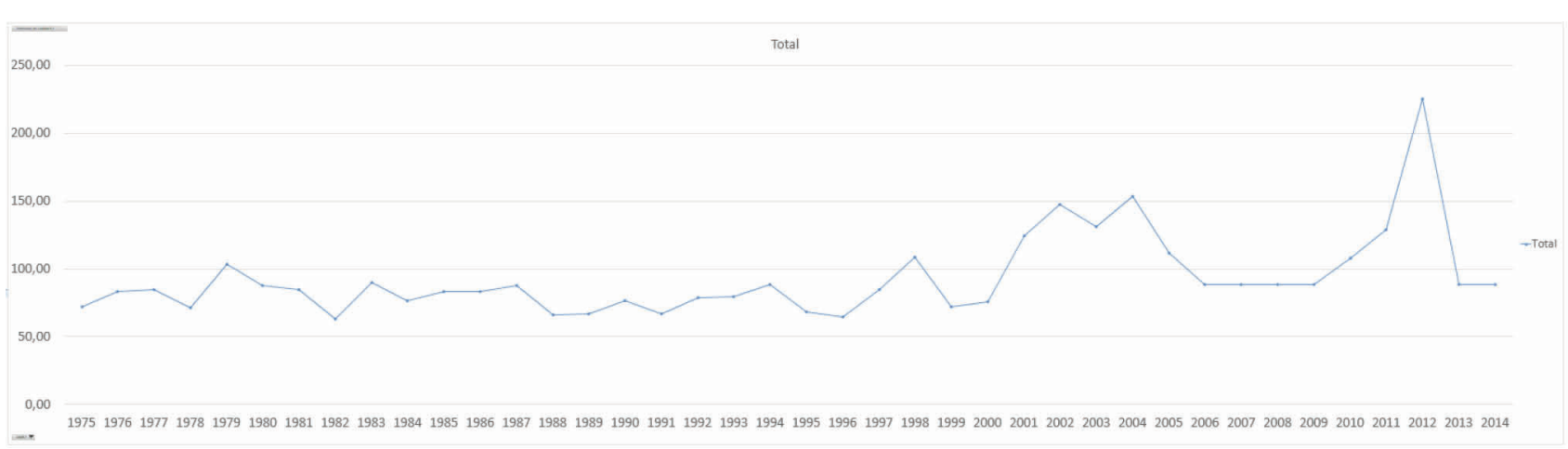

Figura 5. Secuencia de la serie Caudal Puente El Yopal (1975-2014)

Fuente de consulta: Datos IDEAM, completación de datos y procesamiento por parte del investigador.

Este gráfico presenta de manera preliminar que no existe tendencia en la serie por lo cual de manera intuitiva se afirmar que existe estacionariedad en media y en varianza. Por otra parte, se observan picos que evidencian estacionalidad la cual, es una característica propia de la serie y se corrobora mediante los factores estacionales relacionados en la tabla No 6. 
Tabla 6. Factores estacionales de estudio

\begin{tabular}{|c|c}
\hline Período & Factor estacional (\%) \\
\hline 1 & 33,3 \\
\hline 2 & 39,6 \\
\hline 3 & 45,2 \\
\hline 4 & 52,9 \\
\hline 5 & 68,6 \\
\hline 6 & 88,2 \\
\hline 7 & 106,3 \\
\hline 9 & 121,0 \\
\hline 10 & 135,9 \\
\hline 11 & 151,3 \\
\hline 12 & 166,3 \\
\hline
\end{tabular}

Fuente de consulta: Datos IDEAM, completación de datos y procesamiento por parte del investigador.

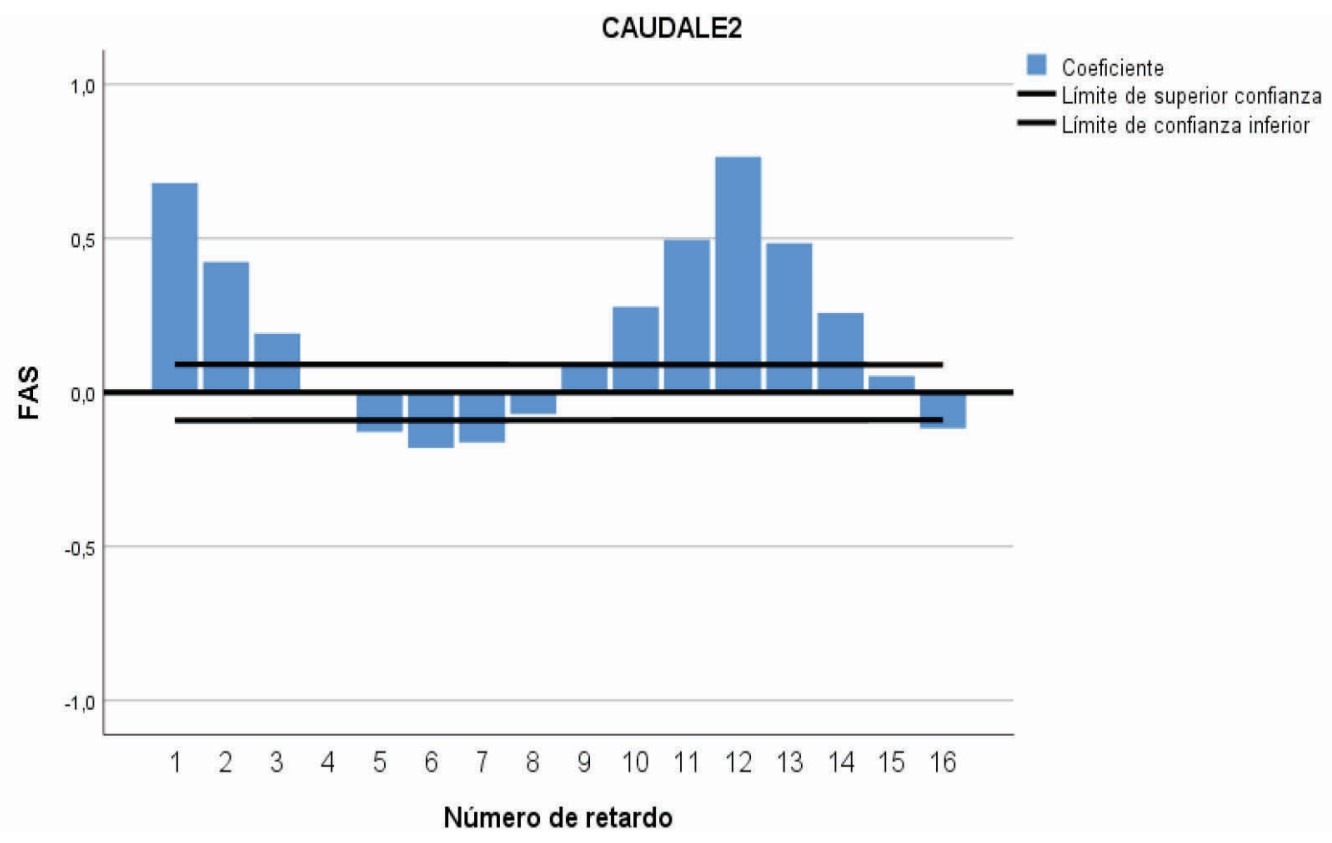

Figura 6. Función de autocorrelación. Fuente: Autores

Con el fin de corroborar la existencia de es- lentamente, la estacionariedad está presente tacionariedad, se realiza análisis al grafico en la serie.

No 5 el cual, muestra que, al no decrecer 
Tabla 7. Descripción del modelo de pronostico

\begin{tabular}{l|l|l|l} 
& \multicolumn{2}{|c}{ Tipo de modelo } \\
\hline ID de modelo & CAUDALE2 & Modelo_1 & ARIMA $(1,0,0)(0,1,1)$ \\
\hline
\end{tabular}

Fuente de consulta: Datos IDEAM, completación de datos y procesamiento por parte del investigador

Utilizando el módulo para pronósticos del Software IBM SPSS Statistics el cual, "es el principal software estadístico que ofrece técnicas de recolección de datos y analítica predictiva para solucionar múltiples problemas empresariales y de investigación. Brinda varias técnicas, que incluyen pruebas de hipótesis lo que facilita la gestión de los datos, la selección y la ejecución de análisis y el intercambio de resultados, así como análisis multivariados y de sobrevida" (Statistics, IBM COLOMBIA S.A., 2018) y de acuerdo a las características de la serie, se obtiene un Modelo Estacional Autoregresivo Integrado de Medias Móviles ARIMA $(p, d, q)(P, D, Q)$.

Tabla 8. Prueba de Ljung-Box Q(18)

\begin{tabular}{|l|c|c|c|}
\hline \multirow{2}{*}{ Modelo } & \multicolumn{3}{c}{ Ljung-Box Q(18) } \\
\hline & Estadístico & DF & Sig. \\
\hline CAUDALE2-Modelo_1 & 12,444 & 16 &, 713 \\
\hline
\end{tabular}

Fuente de consulta: Datos IDEAM, completación de datos y procesamiento por parte del investigador

De acuerdo a la tabla No 8, se evidencia que, que son independientes indicándo que el moal evaluar la independencia de los residuales, delo de pronostico seleccionado es robusto. estos no se encuentran correlacionados dado

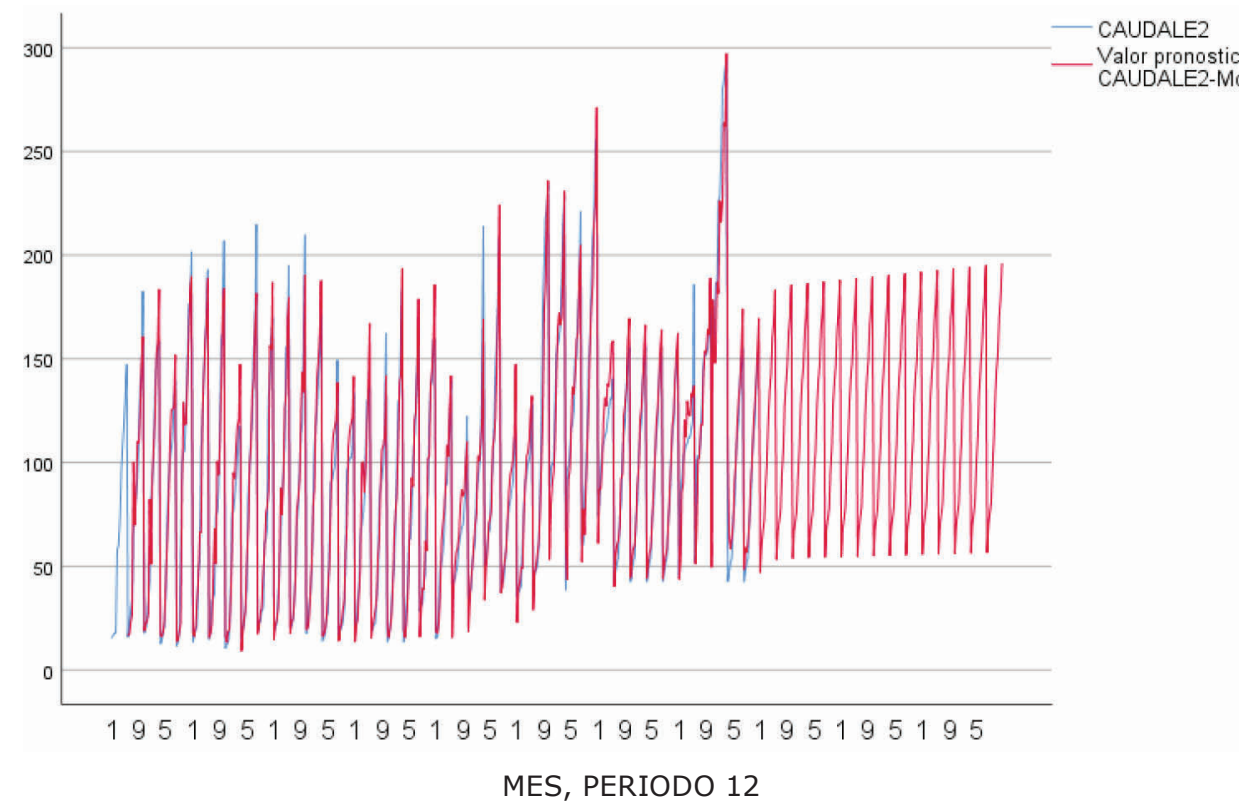

Figura 7. Evaluación del modelo de pronostico

Fuente de consulta: Datos IDEAM, completación de datos y procesamiento por parte del investigador. 
Tabla 9. Estadísticos de prueba

\begin{tabular}{l|c}
\multicolumn{2}{c}{ CAUDALE2 } \\
\hline U de Mann-Whitney & 108003,00 \\
\hline W de Wilcoxon & 217749,00 \\
\hline$Z$ &,- 365 \\
\hline Sig. asintótica(bilateral) &, 715 \\
\hline
\end{tabular}

Fuente de consulta: Datos IDEAM, completación de datos y procesamiento por parte del investigador.

Realizado el pronóstico, se realiza prueba de Mann Whitney con el fin de determinar si existen diferencias significativas entre los datos iniciales y los pronosticados, evidenciándose que debido a que su significación se encuentra por encima del 5\%, no existen diferencias significativas entre las series, por lo tanto, se puede afirmar que el modelo utilizado es eficiente para el pronóstico.
Con el pronostico final se procede a realizar análisis exploratorio de datos para cada mes, donde se obtuvo los estadísticos descriptivos básicos, que permitieron evaluar preliminarmente la serie pronosticada identificando el promedio del caudal el cual, tiende a aumentar anualmente, mientras que, promediando el caudal de cada mes durante los quince años pronosticados, los meses con mayores caudales tienden a ser los del tercer trimestre de cada año. Tabla 10 (Anexo).

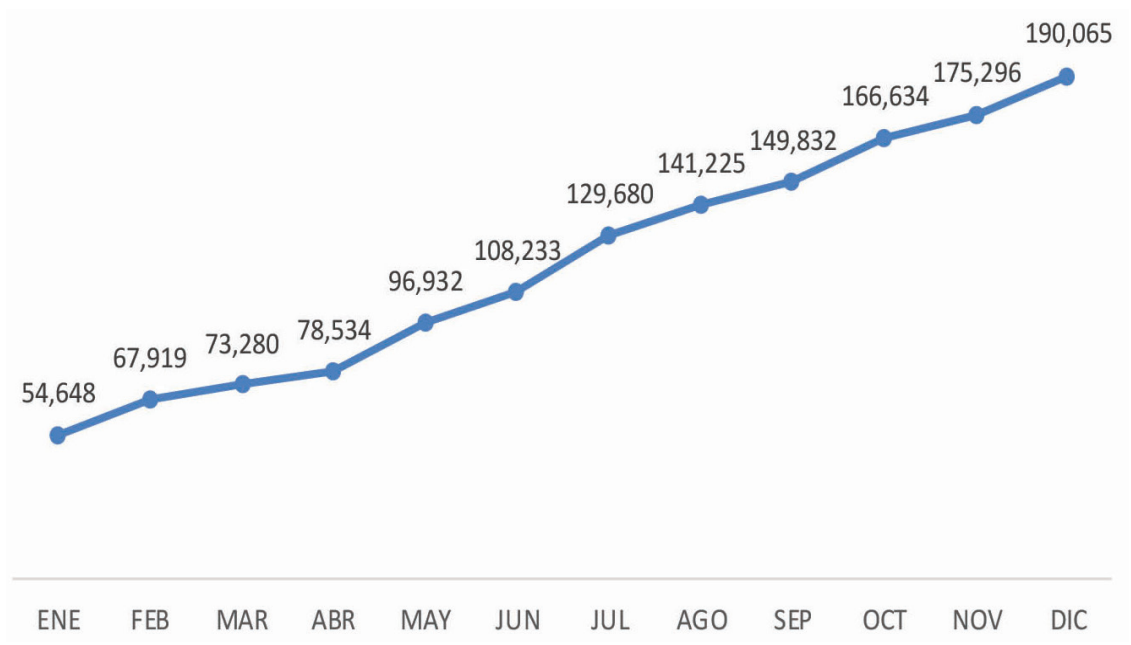

Figura 8. Promedio del valor pronosticado según mes para los 15 años

Fuente de consulta: Datos IDEAM, completación de datos y procesamiento por parte del investigador. 


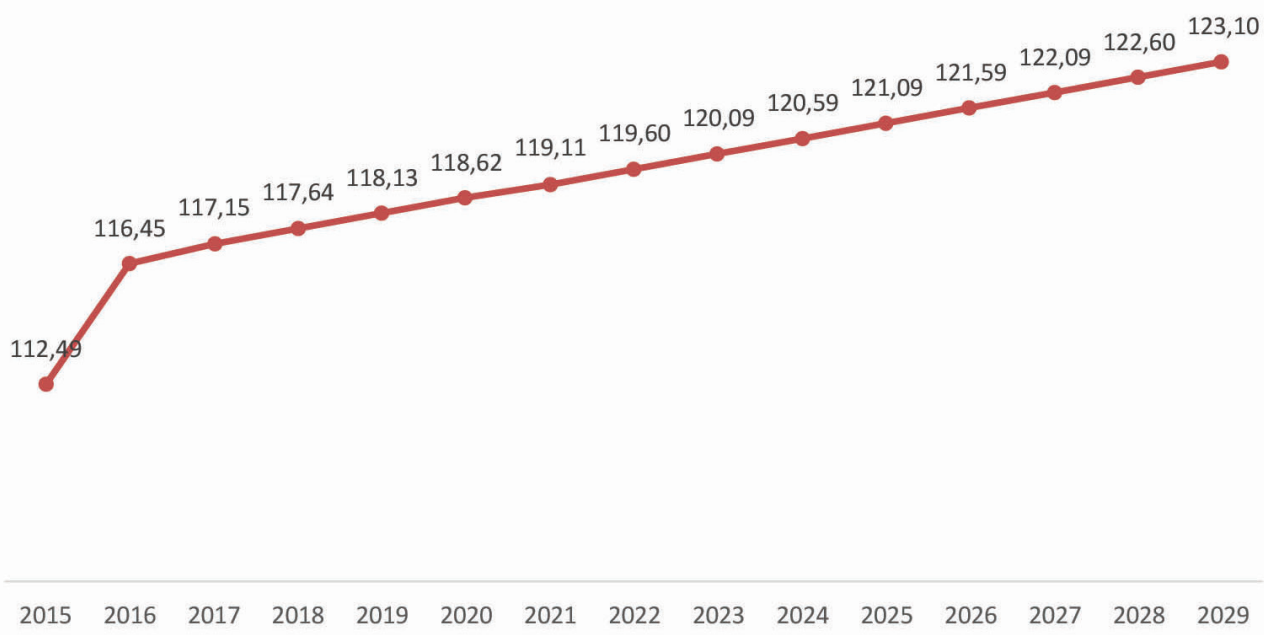

Figura 9. Promedio del valor pronosticado según mes para los 15 años Fuente de consulta: Datos IDEAM, completación de datos y procesamiento por parte del investigador.

\section{CONCLUSIONES}

Los datos aquí presentados servirán como fundamento teórico para la comunidad en general y a entes gubernamentales quienes, de acuerdo a su criterio, puedan tomar decisiones en pro del desarrollo de la región teniendo en cuenta, que son datos de caudales que posiblemente puedan ocurrir en la región y que podrían o no afectar los sectores afines a esta área como lo son el agro y ganadero además de la calidad de vida de la población del municipio El Yopal principalmente.

Se observa que el comportamiento del caudal en la cuenca media del Río Cravo Sur es uniforme mostrando ciclos anuales de estacionalidad presentados en las estaciones hidrometeorológicas ubicadas en esta zona de estudio.

En el análisis exploratorio realizado a los datos iniciales se obtuvo información descriptiva suficientemente útil sobre el comportamiento general de los datos, los cuales fueron corroborados con los resultados obtenidos en los análisis confirmatorios; cabe destacar también que este es un paso necesario para realizar cualquier tipo de investigación ya que, permite tener una visión descriptiva de los datos con ayuda de los estadísticos básicos, los gráficos y pruebas de normalidad. hasta el año 2014 pero ello no implica que los datos obtenidos sean cien por ciento reales por lo cual, podría ocurrir un evento anormal de caudal en la zona llegando a niveles no vistos antes debido a la constante variación del clima en los últimos años, es recomendable que la comunidad en general solicite a las entidades locales (alcaldías, corporaciones regionales ambientales entre otras), estudios más especializados con el fin de prevenir eventos no deseados.

Es importante continuar realizando investigaciones de este tipo, incorporando al análisis otras variables como lo son: temperatura, humedad, precipitación, velocidad del viento, evaporación, evapotranspiración entre otros, con el fin de evaluar el efecto de las mismas sobre los niveles del caudal.

Realizado el análisis del comportamiento de las series históricas, logra evidenciarse que el caudal en algunos sectores de la zona de estudio se encuentra por encima de los caudales aguas arriba y que este aumenta de manera importante principalmente en el sector circundante al municipio de El Yopal en el Departamento de Casanare. 
De acuerdo con el pronóstico realizado, el promedio del caudal en la zona media del río Cravo Sur comprendida entre EL CORREGIMIENTO DEL MORRO - PUENTE LA CABUYA tiende a aumentar anualmente, mientras que, promediando el caudal de cada mes durante los quince años proyectados, los meses con mayores caudales tienden a ser los del tercer trimestre de cada año.

Siempre debe buscarse la posibilidad de realizar análisis con series de datos de tiempo completas con el fin de corroborar la veracidad en el comportamiento de cada variable.

Es claro que se presenta un pronóstico estimados de caudales derivados de datos históricos

\section{LITERATURA CITADA}

Actualización Plan de Ordenación y Manejo de la Cuenca del Rio Cravo Sur Código 3521, pág. 36. Consorcio POMCA 201557

CORPORINIQUIA, CORPOBOYACA, \& UAESPNN. (2007). Plan de Ordenación y Manejo de la Cuenca del Río Cravo Sur. Yopal, Casanare, Colombia.

Hernández Martín, Z. (2012). MÉTOdOS DE ANÁLISIS DE DATOS (APUNTES). España: Servicio de Publicaciones, ed. 519.2.

IDEAM. (2014). Instituto de Hidrología, Meteorología y Estudios Ambientales. Obtenido de http:// www.ideam.gov.co/web/entidad/acerca-entidad

Statistics, I. S. (agosto de 2018). IBM COLOMBIA S.A. Obtenido de https://www.ibm.com/co-es/ products/spss-statistics/details?lnk=hm

\section{AGRADECIMIENTOS}

A la Fundación Universitaria Internacional del Trópico Americano UNITROPICO como universidad que estudia la Orinoquía Colombiana, por la oportunidad de aprendizaje, al docente LEOMAR R. VALECILLOS Licenciado en Ciencias Estadísticas de la Universidad Central de Venezuela por el soporte estadístico para la realización de los análisis.

Conflicto de Intereses Los autores declaran no tener ningún conflicto de intereses 